\title{
Neural Correlates of Feedback Processing in Toddlers
}

\author{
Marlene Meyer ${ }^{1}$, Harold Bekkering ${ }^{1}$, Denise J. C. Janssen ${ }^{1}$, \\ Ellen R. A. de Bruijn ${ }^{2}$, and Sabine Hunnius ${ }^{1}$
}

\begin{abstract}
External feedback provides essential information for successful learning. Feedback is especially important for learning in early childhood, as toddlers strongly rely on external signals to determine the consequences of their actions. In adults, many electrophysiological studies have elucidated feedback processes using a neural marker called the feedback-related negativity (FRN). The neural generator of the FRN is assumed to be the ACC, located in medial frontal cortex. As frontal brain regions are the latest to mature during brain development, it is unclear when in early childhood a functional feedback system develops. Is feedback differentiated on a neural level in toddlers and in how far is neural feedback processing related to children's behavioral adjustment? In an EEG experiment, we addressed these ques-
\end{abstract}

\section{INTRODUCTION}

Monitoring the consequences of one's actions is crucial for successful learning. Feedback informs humans about the consequences of their behavior. The cognitive process of monitoring action outcomes based on external signals is called "feedback processing" (Müller, Möller, Rodriguez-Fornells, \& Münte, 2005). In early childhood, when milestones of motor, cognitive, and social development expand young children's abilities of interacting with their environment, feedback processing is especially important for learning. It enables young children to evaluate their daily actions and to learn how to adjust future actions (Bohlmann \& Fenson, 2005). Yet, little is known about how toddlers process feedback in early childhood and how this guides their behavior.

In adults, electrophysiological studies have demonstrated differential brain activity for feedback indicating correct and incorrect outcomes (Walsh \& Anderson, 2012). More specifically, in the ERP locked to feedback onset, a larger negative deflection is found about 200 300 msec after feedback indicating incorrect outcomes (see Walsh \& Anderson, 2012, for a review). This neural marker of feedback processing is called the feedbackrelated negativity (FRN) or medial frontal negativity and has been associated with learning and adaptive behavior (Cohen \& Ranganath, 2007; Gehring \& Willoughby, 2002; Holroyd \& Coles, 2002; Miltner, Braun, \& Coles, 1997).

\footnotetext{
${ }^{1}$ Radboud University Nijmegen, ${ }^{2}$ Leiden University
}

tions by measuring the brain activity and behavioral performance of 2.5-year-old toddlers while they played a feedback-guided game on a touchscreen. Electrophysiological results show differential brain activity for feedback with a more negative deflection for incorrect than correct outcomes, resembling the adult FRN. This provides the first neural evidence for feedback processing in toddlers. Notably, FRN amplitudes were predictive of adaptive behavior: the stronger the differential brain activity for feedback, the better the toddlers' adaptive performance during the game. Thus, already in early childhood toddlers' feedback-guided performance directly relates to the functionality of their neural feedback processing. Implications for early feedback-based learning as well as structural and functional brain development are discussed.

Findings based on fMRI research suggest that the ACC, a medial prefrontal brain structure, plays a key role in feedback processing (Holroyd et al., 2004; Ridderinkhof, Ullsperger, Crone, \& Nieuwenhuis, 2004). During brain development, ACC shows a protracted maturation compared with more posterior brain regions (Casey, Tottenham, Liston, \& Durston, 2005; Gogtay et al., 2004). Besides structural maturation, ACC also shows developmental change in functionality (e.g., for processing the informative value of feedback) until late adolescence (Crone, Zanolie, van Leijenhorst, Westenberg, \& Rombouts, 2008; Crone, Somsen, Zanolie, \& van der Molen, 2006). Together, these findings suggest an immaturity of brain structures and functions related to feedback processing in early childhood. Still, in the third year of life, feedback-guided learning is evident in toddlers' motor and cognitive behavior. At this age, children are sensitive to external feedback on their actions (Bohlmann \& Fenson, 2005). This raises the question whether toddlers show differential brain responses to feedback and in how far feedbackrelated brain responses might be related to adaptive behavior. Given that frontal brain regions are still in a developmental state, interindividual variation in neural differentiation might be linked to behavioral measures of feedback processing (i.e., adaptive behavior).

We conducted an ERP study in which 2.5-year-old toddlers played a simple feedback-guided game. During each trial of the game, toddlers went first through a gambling phase (i.e., the chances for a correct response were at chance) and an informed phase in which the 
toddlers could make use of the feedback provided during the gambling phase to guide their performance accordingly. Our hypotheses were twofold: First, if toddlers can differentiate feedback information, we would expect them to show a feedback-locked ERP with a more pronounced negativity following incorrect outcomes, as described in the adult literature (Holroyd \& Coles, 2002). Second, we hypothesized that the degree of neural feedback differentiation between correct and incorrect outcomes would reflect the functionality of the developing feedback processing system (Hämmerer, Li, Müller, \& Lindenberger, 2010; Cohen \& Ranganath, 2007). Hence, we expected the FRN difference for correct and incorrect outcomes to be related to children's feedback-guided performance during the informed phase of the game.

\section{METHODS}

\section{Participants}

Nineteen 30-month-old toddlers were recruited from a database of families who had signed up for participation in child research at the Baby Research Center in Nijmegen, the Netherlands. Parents gave written consent after receiving information about the methods and purpose of the study. The families visited the laboratory for a session of approximately 1-hr duration. They received a small present or monetary compensation for their participation. The final sample consisted of 12 toddlers (seven girls; mean age = 30.68 months, $S D=0.19$ months). Another seven toddlers were tested but were excluded from final analyses for one of two reasons. One child was unwilling to wear the EEG cap. The other six participants did not provide a sufficient amount of artifact-free EEG trials that were also behaviorally valid trials (for details about the inclusion criteria, see Data Analysis).

\section{Task and Stimuli}

The toddlers performed a computerized task that was implemented using a touch screen interface. The aim of the task was to find two cards with identical pictures. The stimulus set consisted of cards with 11 different animals, which toddlers are typically familiar with and able to name (e.g., a cow, a lion, a pig). Each trial started out with three cards, presented on the screen in a vertical configuration, with the middle card always defined as the "reference" card. The upper and the lower cards showed an animal that was either identical to or different from the animal shown in the middle (see Figure 1A). In the "initial phase," the toddlers were asked to identify the card that was identical to the reference card and to indicate their choice by pressing the respective card on the screen. The initial phase was aimed to (1) ensure task understanding and (2) prevent children from deliberately choosing a wrong card out of curiosity about the identity of a hidden card in the subsequent phases. Upon pressing the matching card, both the upper and the lower card turned face-down, leaving the animal pictures hidden from sight. (Note that, at this point, pressing the mismatching card, reference card, or any other location on the screen would not trigger any effect.) Once the upper and lower card were turned face-down, the "shuffle phase" commenced. Here, the upper and lower card moved in a swift, circular pattern around the stationary reference card for approximately $2 \mathrm{sec}$ (see Figure 1B). The shuffling was quick, and the final pattern random such that it was impossible to track the cards' movements and derive accurate predictions about their ending positions. The cards ended up in a horizontal configuration (see Figure 1C). The "first turn (gambling phase)" followed. It was the child's task to try to find the card depicting the animal identical to the reference card by pressing either the left or the right card. Upon pressing,
Figure 1. Exemplar sequence of events in a single trial. (A) "Initial phase": Participants were instructed to select the matching card, which contained the picture that was identical to the reference (middle) card. (B) "Shuffle phase": The matching card and mismatching card were shuffled (face-down). The experimenter explained: "Now the animals will hide." (C) "First turn (gambling phase)": the experimenter asked the child: "Where is the other [animal name]?" referring to the identity of the animal shown on the reference card. The participant guessed whether the matching card was located on the left or the right (shown is a situation in which the card on the right was chosen). (D) The picture of the chosen card was revealed (shown is an example of an incorrect first turn, i.e., the mismatching card was found). The nature of the outcome (correct/incorrect) was unpredictable. (E) "Second turn (informed phase)": The participant made a second attempt to find the matching card. (F) On the basis of the knowledge gained in the first turn, a correct outcome could, theoretically, be obtained in each second turn.

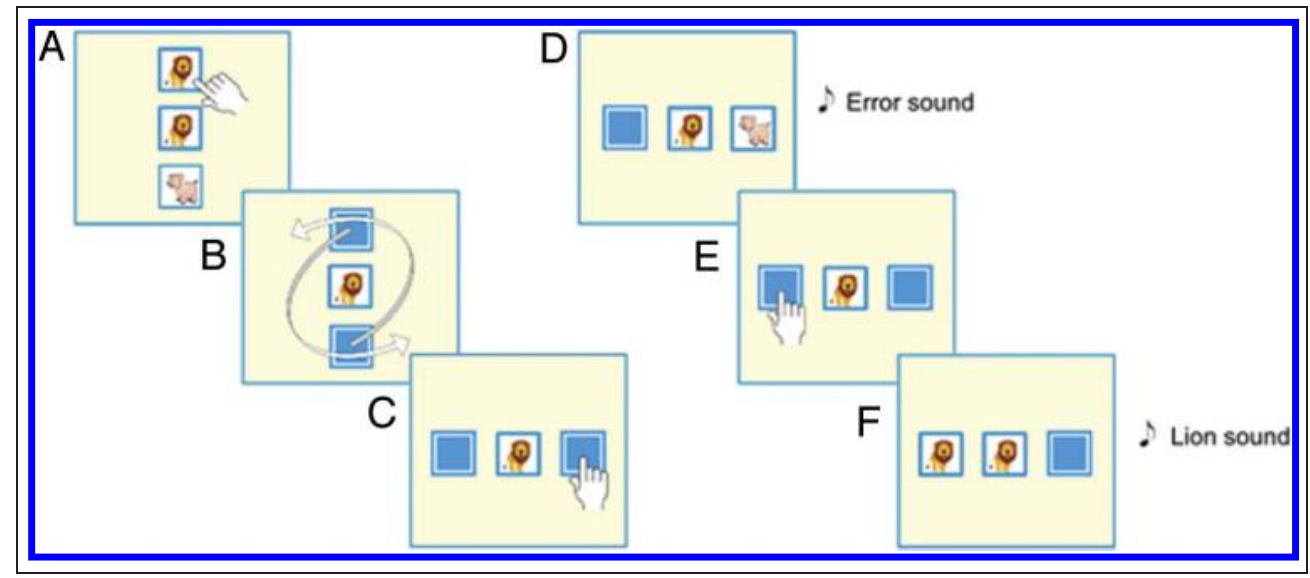


the chosen card turned face-up and revealed its picture (see Figure 1D). The picture presentation served as visual feedback as to whether the child chose the correct or incorrect card (i.e., the outcome of the first turn was correct when the card that matched the reference card was found and incorrect when the mismatching card was found). In case the child had found the correct card, an additional auditory stimulus (the corresponding animal sound) followed $1000 \mathrm{msec}$ after the picture presentation of the card. If the incorrect card had been found, a low-pitched beep sound followed after $1000 \mathrm{msec}$. The duration of the auditory stimuli was approximately $1 \mathrm{sec}$. Simultaneous to the auditory stimulus (i.e., delayed with respect to the visual picture appearances), the experimenter also gave verbal feedback: "yes, you have found it," in case of the matching card and "no, that's not the one" in the case of the mismatching card. Note that the auditory and verbal feedback served only to encourage the children and was not the feedback assessed in analyses. Instead, the feedback-locked ERPs were time-locked to the visual feedback, that is, the appearance of the picture on the back of the chosen card. The chance of finding the matching card in the first turn was $50 \%$ as we provided two options to choose from and no additional information as to which card was the correct card. Thereby, approximately half of the trials had correct (and half incorrect) action outcomes on the first turn. Next, in the "second turn (informed phase)," the chosen card (independent of its correctness) turned face-down again while remaining at the same location, so that both the left and the right animal pictures were hidden from sight once more. This last phase of each trial followed an identical procedure to the first turn (i.e., the experimenter asked where the animal identical to the reference picture was hidden and the child could choose either the left or the right card; see Figure 1E, F). The crucial difference between the first and the second turn was the predictability of their outcome. On the basis of the outcome of the first, gamble choice, toddlers could infer the location of the matching card in the second turn. The rationale to include the second turn was to ensure that toddlers understood the goal of the game and the feedback in the gambling phase and to test whether toddlers could make use of the feedback in their subsequent performance. Thus, children's choice behavior in the second turns (informed phase) allowed us to investigate whether they adjusted their actions according to the feedback information obtained in the first turns. Note that each trial contained a second turn, that is, the occurrence of the second turn was fixed and independent of the outcome of the first turn.

\section{Procedure}

The experiment took place in an electrically shielded room. Before starting the testing session, parents were instructed not to interfere during the measurement and to minimize any interaction with their child during the experiment. Throughout the session, the toddlers sat on the lap of a parent. After preparing the EEG cap, the experimenter explained the goal of the game (finding the matching animal) and demonstrated two trials. The parent was instructed not to actively engage in the game or influence their child's behavior in any way. Toddlers could play a total of 55 trials (each consisting of two consecutive turns), taking approximately 25 min of playing time. There were five blocks in which each of the 11 different animals appeared on the reference card once. The order of reference animals within blocks and the identity of the mismatching animal were randomized.

\section{EEG Recording}

The experiment was controlled by Presentation Software (Neurobehavioral Systems; www.neuro-bs.com). In addition to recording the card choices and their outcomes, the children's behavior was videotaped. EEG was recorded with a sampling rate of $500 \mathrm{~Hz}$ from 32 scalp sites according to the International 10-20 system, using active Ag/AgCl electrodes (ActiCAP), Brain Amp DC, and Brain Vision Recorder software (Brain Products GmbH, Germany). The data were filtered on-line with a low cutoff at $0.016 \mathrm{~Hz}$ and a high cutoff at $125 \mathrm{~Hz}$. Electrode impedances were kept below $25 \mathrm{k} \Omega$. The left mastoid was used as the on-line reference. Off-line, the data were rereferenced to the averaged mastoids.

\section{Data Analysis}

Behavioral performance was analyzed in terms of the percentage of correct outcomes on second turns. A video analysis was performed to exclude invalid trials, which were identified using the following criteria: (1) First or second turns in which the child did not attend to the screen during the turning of the cards (feedback presentation) and (2) second turns that were preceded by distraction (i.e., when the child was distracted from the information provided by the first turn).

Feedback-locked ERPs were obtained by creating EEG segments between 200 msec before and 1600 msec after feedback onset (i.e., the appearance of the picture on the card that was chosen in the first turn). As in previous studies on the FRN, a 200-msec prefeedback period was used for baseline correction (e.g., Hajcak, Moser, Holroyd, \& Simons, 2006; Holroyd, Hajcak, \& Larsen, 2006). A 30-Hz low-pass filter and filter padding were applied. Upon visual inspection of the feedback-locked ERPs, trials containing artifacts were rejected from further analysis. For each participant, an averaged feedbacklocked ERP was obtained per outcome category: (1) correct first turn and (2) incorrect first turn. The final data set was restricted to participants who provided at least 10 behaviorally valid trials that were free of EEG artifacts for each of the two outcome categories. On average, the 
total number of excluded trials was 33.78\% ( $S D=$ $16.61 \%$ ) for trials with correct first turns and 30.92\% $(S D=15.66 \%)$ for trials with incorrect first turns. The mean number of trials included in the statistical analysis was $15.75(S D=5.63$, range $=10-26)$ for correct first turns and $16.17(S D=5.42$, range $=10-26)$ for incorrect first turns.

As a measure of the FRN, we calculated the mean amplitude within a 100-msec time window. To define the FRN time window, we determined the latency of the peak amplitude in the difference wave, which was calculated by subtracting the grand average of the activity elicited in response to correct first turns from the activity elicited in response to incorrect first turns (cf. Holroyd \& Coles, 2002). We centered the 100-msec time window around the peak of the negative deflection in the difference wave, which was 372 msec after feedback onset. Subsequently, this time window was applied in the analysis of the feedback-locked ERPs for correct and incorrect outcomes, separately. Note that the time window of analysis was data-driven rather than based on previous reports in adults and older children. As ERP components are known to shift in latency with increasing age (Nelson \& McCleery, 2008), the time window of analysis could not be defined a priori. In contrast, the selection of electrodes for the statistical analysis was based on the adult FRN literature, which has generally identified the frontal midline electrodes Fz, FCz, and $\mathrm{Cz}$ to show the strongest effect (Mai et al., 2011; Bellebaum, Polezzi, \& Daum, 2010; Boksem, Kostermans, \& de Cremer, 2010; Fukushima \& Hiraki, 2009; Bellebaum \& Daum, 2008; Holroyd \& Krigolson, 2007; Gehring \& Willoughby, 2002). Because ACC has been suggested as the cortical source of the FRN (Bellebaum \& Daum, 2008; Debener et al., 2005; Gehring \& Willoughby, 2002; Miltner et al., 1997), we expected a similar frontal midline topography for young children. To additionally verify this a priori selection of electrodes, we subsequently plotted a topographic map of the difference wave at the maximum peak of the difference between correct and incorrect first turns.

Statistical analyses were performed using SPSS 15.0 for Windows (SPSS Inc., Chicago, IL). To test the effect of feedback (correct outcome versus incorrect outcome in the gambling phase), the mean amplitudes of the feedbacklocked ERPs were entered as within-subject factors in a two-way repeated-measures ANOVA. Electrode (Fz, FCz, and $\mathrm{Cz}$ ) was included as an additional within-subject factor. The assumption of sphericity was tested, and corrected values are reported where appropriate. After visual inspection of the feedback-locked ERPs (see Figure 2), we conducted an additional analysis of the ERP data to investigate whether the FRN amplitude difference was related to the latency difference between correct and incorrect outcome waveforms. Possibly larger latency differences between the two types of feedback-locked ERPs (correct and incorrect outcome) could lead to larger amplitude differences in the FRN time window. Consequently, the FRN

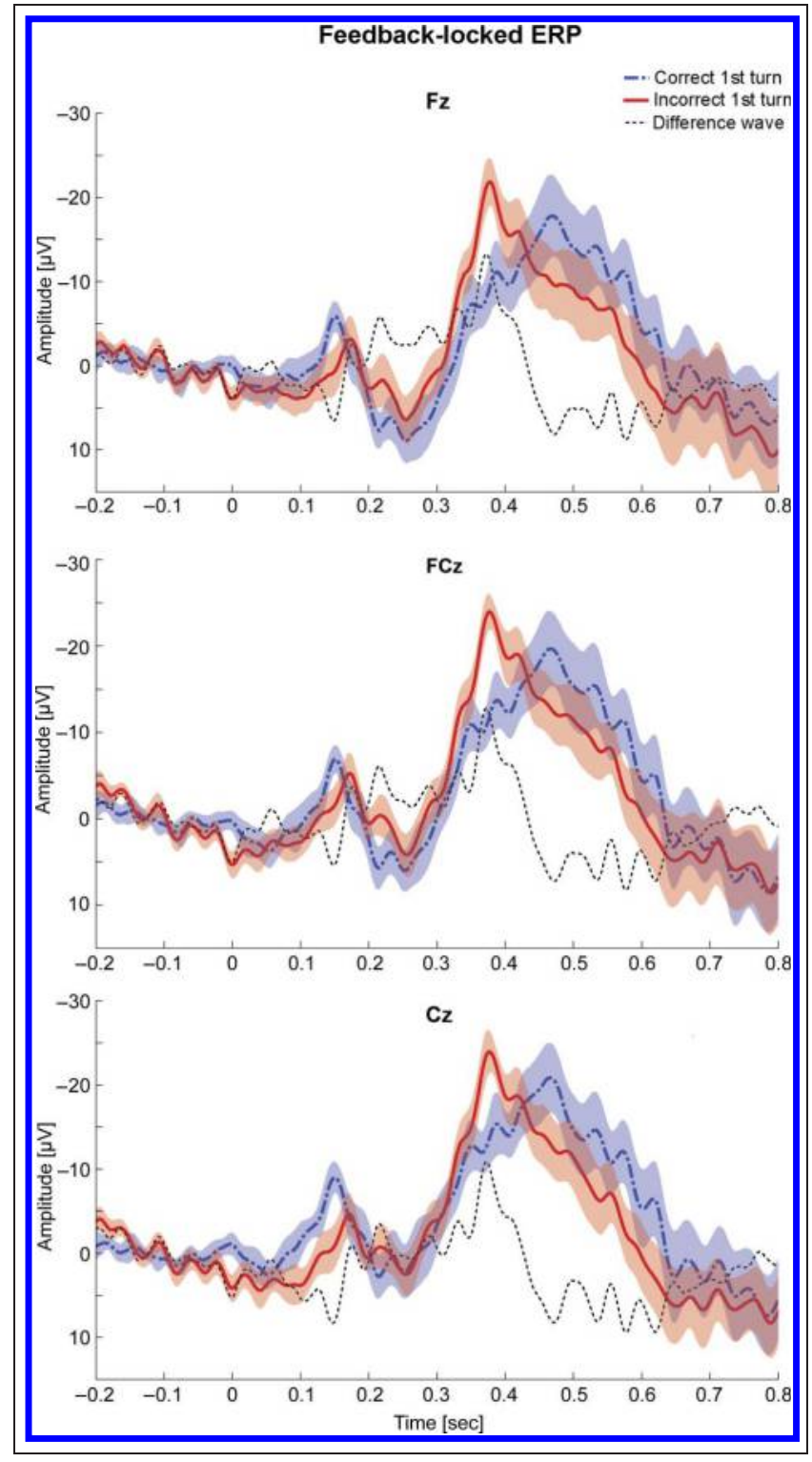

Figure 2. Feedback-locked ERPs for correct (blue, dashed line) and incorrect (red, solid line) first turns. The dotted black line illustrates the difference between correct and incorrect first turns. Mean $\pm 1 S E$ are plotted in accordingly shaded areas for electrode Fz, FCz, and Cz.

amplitude difference would reflect differences in processing speed rather than a distinction in amplitude as found typically in the adult FRN literature (Walsh \& Anderson, 2012). To test whether latency differences were closely related to the FRN amplitude difference, we correlated individual latency differences (correct-incorrect) of the negative peak (between 0 and $1000 \mathrm{msec}$ locked to feedback onset) with individual amplitude differences in the FRN time window (correct - incorrect). To further test for possible differences in peak latency between conditions, we conducted a repeated-measures ANOVA with the factors Feedback (correct vs. incorrect) and Electrode (Fz, $\mathrm{FCz}$, and $\mathrm{Cz}$ ) on the latency values of the negative peak (between 0 and 1000 msec locked to feedback onset). 
In addition to analyzing the electrophysiological data only, we performed a correlational analysis assessing any relation between the electrophysiological data and the behavioral performance. As a measure of electrophysiological differentiation between feedback on correct and incorrect outcomes, the amplitude for incorrect first turns was subtracted from the amplitude for correct first turns. Thus, a larger, positive number on this measure reflected a larger degree of differentiation. We reasoned that the ability to distinguish correct and incorrect outcomes in the gambling phase (as reflected by the differential EEG response) would enable the use of this feedback information to perform second turns (i.e., the informed phase) correctly. Therefore, the measure of electrophysiological differentiation was entered in a correlation analysis to determine its association with behavioral performance (defined as the percentage of correct second turns). After validating the normality assumption for the Pearson's $r$, the correlation coefficient was determined for the average of $\mathrm{Fz}, \mathrm{FCz}$, and $\mathrm{Cz}$.

\section{RESULTS}

\section{Behavioral Data}

Of the 12 toddlers whose data were included in the statistical analyses, six completed all 55 trials. On average, the number of completed trials was $47.58(S D=10.03)$, ranging between 26 and 55 . The mean percentage of correct first turns was 50.42\% (SD = 7.78\%), as expected given the gambling nature of the first turns. Of the second turns, on average $81.74 \%$ ( $S D=12.92 \%)$ were performed correctly. Overall, toddlers performed significantly above chance on the second trials of the game, $t(11)=8.511$, $p<.001$.

\section{ERP Data}

In the feedback-locked ERP, a negative deflection started to emerge approximately $250 \mathrm{msec}$ after the onset of feedback. For feedback representing an incorrect first turn, the negative deflection reached its peak at approximately $370 \mathrm{msec}$. A negative deflection for correct outcomes reached its maximum amplitude later, at approximately 470 msec (see Figure 2). The repeated-measures ANOVA testing the effect of Feedback (correct vs. incorrect action outcome) and Electrode revealed a significant main effect on Feedback outcome, $F(1,11)=6.82, p=.024$, with the amplitude of the negative deflection being more negative for feedback on incorrect first turns $(M=-16.94, S D=$ 2.23) than for feedback on correct first turns $(M=-10.48$, $S D=2.55)$. A main effect of Electrode was marginally significant, $F(2,22)=3.27, p=.057$, but no significant effect was found for the interaction between Feedback and Electrode, $F(2,22)=1.58, p=.23$. Closer inspection of the marginally significant main effect of Electrode revealed that amplitudes were most negative for FCz $(M=$
-15.38) and least negative for Fz $(M=-11.44)$. Additionally, a topographic map of the feedback effect (incorrect correct) at the peak onset of the difference wave (372 msec) illustrates the expected frontocentral scalp distribution typical for the FRN (see Figure 3).

Correlating individual FRN amplitude differences with individual latency differences did not show any significant relation $(r=.22, p=.50)$ between the two measures. Thus, the results render it unlikely that larger differences in latency between processing the correct and incorrect outcome are linked to larger FRN amplitude differences.

In addition, the repeated-measures ANOVA testing for differences in peak latencies indicated no significant differences, neither for the individual factors Feedback (correct: $M=449 \mathrm{msec}, S E=38$; incorrect: $M=420 \mathrm{msec}$, $S E=19)$ and Electrode (Fz: $M=456 \mathrm{msec}, S E=36$; FCz: $M=434 \mathrm{msec}, S E=28$; Cz: $M=413 \mathrm{msec}, S E=16)$, nor for the interaction of these factors (all $p>.05$ ).

\section{Correlation Analysis}

Furthermore, we investigated the relation between the differential ERP responses to feedback (correct vs. incorrect action outcome) and the individual task performance. The correlation analysis revealed a statistically significant, positive correlation between the differential ERP responses and task performance $(r=.70, p<$ $.05)$. More specifically, a larger electrophysiological difference between correct and incorrect first turns was

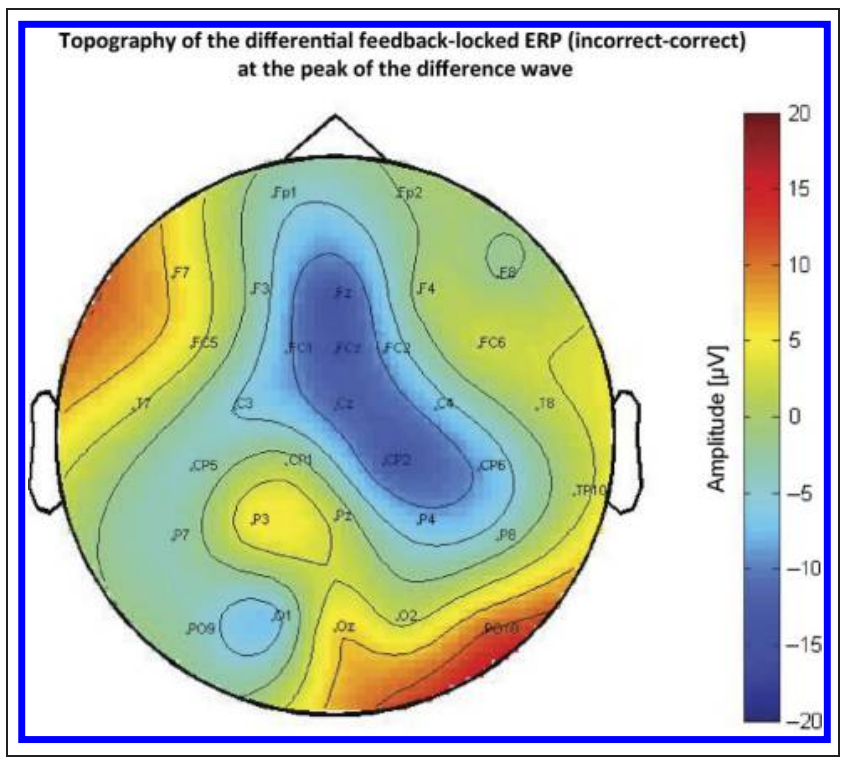

Figure 3. Topographic map of the feedback effect (incorrect - correct) at the peak of the difference wave $(372 \mathrm{msec})$. Note that because of rejection of noisy channels in some of the toddlers, the electrodes represent pooled data of a varying number of toddlers. The range of participants included per channel is 5 (min) to 12 (max). In addition, data are excluded in which the amplitude exceeded $\pm 100 \mathrm{mV}$. 


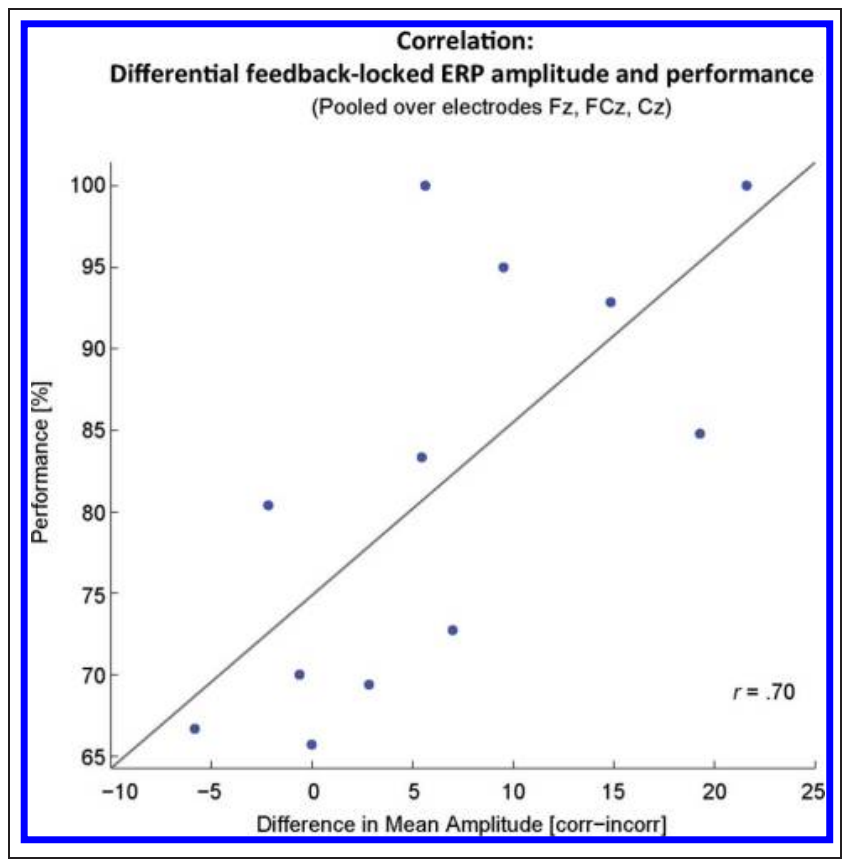

Figure 4. Correlation between the differential feedback-locked ERP amplitude and behavioral performance on second turns pooled over electrodes $\mathrm{Fz}, \mathrm{FCz}$, and $\mathrm{Cz}$.

associated with better behavioral performance on second turns. The result of the correlation analysis are illustrated in Figure 4.

\section{DISCUSSION}

In this study, we explored the neural correlates of feedback processing and behavioral adjustment of 2.5-yearold toddlers during a feedback-guided task. There are two main findings: First, toddlers showed a feedbacklocked ERP with a more pronounced negativity following incorrect outcomes providing the first neural evidence for feedback processing in toddlers. Second, the degree of neural feedback differentiation was related to the degree of behavioral adaptations and thus reflected the functionality of the developing feedback processing system.

\section{Characteristics of the Feedback-related Brain Potentials in Toddlers}

Our toddler sample showed a differential brain response to feedback indicating correct and incorrect action outcomes, which is analogous to previous findings with adults (Walsh \& Anderson, 2012). Consistent with a neural generator in ACC, the frontocentral scalp distribution of the toddlers' feedback effect also resembles the topography seen in adults (Walsh \& Anderson, 2012). Still, as expected on the basis of previous developmental ERP studies (cf. De Haan, 2007), the waveforms in toddlers differed slightly from the typical feedbacklocked waveforms found in adults (e.g., in latency and amplitude). For instance, the peak amplitude of the FRN was delayed for the toddlers (372 msec after stimulus onset in our toddler sample compared with $250 \mathrm{msec}$ in adults; Holroyd et al., 2006). This latency shift is in accordance with indications of delayed neural processing as found with developmental populations in general (e.g., Csibra, Kushnerenko, \& Grossmann, 2008). Delayed neural processing has, for instance, been associated with protracted development of neuronal myelination in the cortex (e.g., Casey et al., 2005). Visual inspection of the toddlers' feedback-locked waveforms might also suggest that amplitude differences in the FRN window result from latency differences between the peaks for correct and incorrect outcomes (see Figure 2). However, statistical tests did not provide any support for this possibility, as there was no significant correlation between the latency and amplitude differences in the ERP and no difference in latency between conditions. Moreover, our findings are in line with the findings of Mai and colleagues (2011), who showed a feedback-locked ERP peaking at $370 \mathrm{msec}$ in 4- to 5-year-olds. Although their results on ERP latency were coherent with our findings, Mai and colleagues (2011) were unable to find indications of a distinction with respect to outcome correctness. This discrepancy might be because of the different feedback-reward procedures used in their study (see Mai et al., 2011). Mai and colleagues (2011) used a game in which children's actions were always rewarded with a prize, with the outcomes varying between "nicest" and "least nice" prizes, based on individual rank ordering. It is possible that this difference might have been too subtle to detect a clear distinction of outcome correctness on a neural level. High variability and a low signal-to-noise ratio often mask small effects in infant and toddler EEG data with a typically limited amount of trials (e.g., Hoehl \& Wahl, 2012). Previous research in young and old populations by Marco-Pallarés and colleagues (2011) highlights the role of the signal-to-noise ratio for the detection of a stable FRN. The signal and variability of the current toddler FRN are displayed in Figure 2. The depicted relation of signal to variability supports that despite a relatively low number of trials a reliable FRN can be determine, dependent on the signal-to-noise ratio (Marco-Pallarés et al. 2011).

One possible limitation of the current findings is that the results might have been augmented by the perceptual properties of the feedback stimuli. However, the relation between ERP differentiation and behavioral adaptations renders it unlikely to be the sole source of the findings. In the current setup, we defined the presentation of two matching pictures as a correct action outcome, whereas an incorrect action outcome consisted of two nonidentical pictures. In adults, an ERP component called the mismatch N2, N200, or N270 is elicited by the perceptual conflict of mismatching items (Baker \& Holroyd, 2011; Folstein \& van Petten, 2008). This might have influenced the currently observed pattern of brain potentials (see Baker \& Holroyd, 2011; 
Jia et al., 2007). Analogously, in the domain of processing oddball stimuli, Holroyd (2004) discussed similarities in morphology of the (oddball) N200 and the FRN, two ERP components found to overlap (Holroyd, PakzadVaezl, \& Krigolson, 2008). Although the N200 and the FRN have a similar electrophysiological origin and time course, a recent study by Baker and Holroyd (2011) suggests that they still represent dissociable processes. Although it is not possible to determine the extent to which a perceptual component might have affected the present ERP data, the correlational findings with respect to the relation between brain potentials and behavior support the reflection of feedback processing in the observed toddler ERP in this study. The correlation of brain potentials with behavior indicates that the FRN accounted for at least a significant part of the electrophysiological difference between correct and incorrect outcomes. Importantly, if the differential ERP reflected processing on a perceptual level only, no correlations with feedback-guided performance would be expected. Thus, despite a potential overlap in components, the observed ERP taps significantly into the functionality of the toddlers' feedback processing system.

\section{Functionality of the Developing Feedback Processing System}

The differential feedback-locked ERP and the feedbackguided behavior found in the 2.5-year-olds provide evidence for a generally functional feedback processing system in early childhood. Besides a generally functional feedback system, the correlation between the feedbackrelated measures of neural activity and behavior still indicates variability in the toddlers' feedback processing. Those toddlers who showed a more pronounced difference in their feedback-related brain response to correct and incorrect outcomes were better able to make use of the feedback information in their performance.

So far, only few studies in adults report a link between the FRN and subsequent behavior (Chen, Wu, Tong, Guan, \& Zhou, 2012; Hämmerer et al., 2010; Mathewson, Dywan, Snyder, Tays, \& Segalowitz, 2008; Cohen \& Ranganath, 2007). To our knowledge, Cohen and Ranganath (2007) were the first to report empirical evidence for the hypothesis that neural feedback processing, as indicated by the FRN, is related to feedbackguided behavior. In an EEG study, they showed that feedback-related potentials to losses preceding subsequent behavioral adaptation were more negative than the feedback-related potentials preceding no change in behavior. Similarly, Hämmerer and colleagues (2010) found that stronger differential neural activity to feedback was predictive of better subsequent performance in older adults; a finding consistent with Mathewson and colleagues (2008). This link between the FRN effect and the level of performance in older adults was less consistent in younger individuals between the age of 9 and 30 years (Hämmerer et al., 2010). The correlational findings of the current toddler sample complement these previous findings and offer supporting evidence for a functional relation between FRN and feedbackguided behavior.

Recently, Chen and others (2012) investigated feedback processing in the context of social conformity. Similar to Cohen and Ranganath (2007), Chen and colleagues (2012) show that feedback-locked ERPs are more negative when they precede subsequent behavioral adaptation in contrast to when the ERPs are followed by no behavioral change. In addition, Chen and others (2012) report a relation between the strength of the FRN effect and the level to which the individuals adjusted their behavior to the opinion of others, suggesting feedback processing to be modulated by social factors. Whereas the findings of Cohen and Ranganath (2007) are mainly focused on the neural response to negative feedback and behavioral adaptation, the correlations with behavior found by Chen and others (2012) and Hämmerer and colleagues (2010) as well as the current findings in toddlers take into account the differential neural activity between positive and negative feedback. In the following, we will discuss potential implications of the correlation between the toddlers' differential feedback-locked brain response and their subsequent behavior.

The observed variability might reflect different underlying factors contributing to successful feedback processing and feedback-based learning. Developmental differences, individual differences, and state-related differences within each individual might account for the variability in the toddlers' feedback processing.

First, we will discuss possible contributions of developmental differences to how the toddlers processed the provided feedback. Toddlers might differ from one another with respect to the maturity of their brain structures, which are related to feedback processing (e.g., ACC), as these areas are late to mature in children (Gogtay et al., 2004). Activity in the medial prefrontal areas is involved in the detection and evaluation of feedback in adults (see Walsh \& Anderson, 2012, as review). These areas might be relatively less mature in those toddlers who show less proficient feedback processing and behavioral adjustment compared with toddlers who are better able to adjust their behavior. The immaturity of structures such as ACC might in turn be reflected in functional differences that appear on separate levels of processing. Bush, Luu, and Posner (2000) distinguish two levels of functional processing in ACC: cognitive and affective/emotional processing. Cognitive mechanisms such as attentional control still undergo fundamental developmental changes during early childhood (Rueda, Posner, \& Rothbart, 2004). In a neuroimaging study on attentional control, Casey and colleagues (1997) examined 5- to 16-year-old children and demonstrated a close link between attentional control and structural characteristics of the right ACC. Because the 
feedback-guided task in the current experiment required the allocation of attention to the screen and in particular to the identity of the presented feedback, the functional immaturity of the attentional control system might have contributed to less focused allocation of attention and hence the variability in feedback processing between toddlers. Besides cognitive processing, ACC activity has been associated to the processing and control of affective/ emotional inputs (see Bush et al., 2000, as review). Throughout childhood and adolescence, developmental changes of emotional processing are associated to rostral and ventral areas of ACC and their functional connectivity (see Kelly et al., 2009, for a review). A less mature regulation of emotions might therefore have had an influence on the perceived affective value of the presented feedback and result in the variability between toddlers. Yet, in a study with adolescents and adults, Santesso, Dzyundzyak, and Segalowitz (2011) did not find indications for developmental differences related to emotional regulation but rather individual differences related to feedback sensitivity to account for variability in the FRN.

Besides developmental differences, individual differences in sensitivity to feedback could underlie the toddlers' variability in processing feedback. From research on individuals with depression (Tucker, Luu, Frishkoff, Quiring, \& Poulsen, 2003) and healthy individuals with selfreported feedback sensitivity ratings (Segalowitz et al., 2012; Santesso et al., 2011), it is evident that the amplitude of the FRN differs between individuals in general (see van Noordt \& Segalowitz, 2012, for a review). Accordingly, toddlers who show a smaller differential brain response to feedback on their actions might be, in general, less sensitive to feedback rather than reflecting a relatively immature feedback processing system.

Both developmental and individual differences may further be modulated by state-related changes. Evidence from electrophysiological research with adults demonstrates that motivational states can affect the amplitude of the FRN (Walsh \& Anderson, 2012; Luo, Sun, Mai, Gu, \& Zhang, 2011, as review). Therefore, less optimal performance in the present task might reflect weaker motivation to succeed in the task, rather than a lack of capacity to process the feedback information. If motivational variability underlies the observed variability in the toddlers' feedback-related ERP and behavior, this would suggest at least two implications: On the one hand, it would emphasize the role of motivation for feedbackguided learning in toddlers and thus have implications for educational practice. On the other hand, it would suggest that already at the age of 2.5 years the feedback processing system functions successfully in terms of both feedback evaluation and subsequent adaptive behavior given a high level of motivation. Future research is needed to disentangle the contribution of developmental, individual, and state-related differences to feedback processing in early childhood and to explore the influ- ence of cognition and motivation/emotion in the relation between neural and behavioral aspects of feedback processing in toddlers.

In summary, the current findings are the first to demonstrate neural correlates of feedback processing in 2.5-yearold toddlers. The toddlers showed a more negative deflection in the ERP to incorrect action outcomes, a finding that parallels previous results from adult research. Importantly, the stronger the differential brain response to feedback was, the better the young children adjusted their behavior based on the feedback. These findings imply that feedback differentiation on a neural level is tightly linked to the quality of feedback-guided learning in young children and offer ground for future investigations on the interplay between structural brain maturation and cognitive and emotional factors for feedback processing in early childhood.

\section{Acknowledgments}

We would like to thank the children and parents for participating in this study. Moreover, we thank our laboratory managers Angela Khadar and Margret van Beuningen for their help. We also acknowledge the technical support from Sander Berends and the Technical Support Group at Radboud University Nijmegen. EDB is supported by the Netherlands Organization for Scientific Research (NWO), VIDI grant no. 452-12-005.

Reprint requests should be sent to Marlene Meyer, Donders Institute for Brain, Cognition and Behaviour, Radboud University, P.O. Box 9104, 6500 HE Nijmegen, The Netherlands, or via e-mail:m.meyer@donders.ru.nl.

\section{REFERENCES}

Baker, T. E., \& Holroyd, C. B. (2011). Dissociated roles of the anterior cingulate cortex in reward and conflict processing as revealed by the feedback error-related negativity and N200. Biological Psvchologv, 87, 25-34.

Bellebaum, C., \& Daum, I. (2008). Learning-related changes in reward expectancy are reflected in the feedback-related negativity. European Iournal of Neuroscience, 27, 1823-1835.

Bellebaum, C., Polezzi, D., \& Daum, I. (2010). It is less than you expected: The feedback-related negativity reflects violations of reward magnitude expectations. Neuropsychologia 48 , 3343-3350.

Bohlmann, N. L., \& Fenson, L. (2005). The effects of feedback on perseverative errors in preschool aged children. Lournal of Cognition and Development, 6, 119-131.

Boksem, M. A. S., Kostermans, E., \& de Cremer, D. (2010). Failing where others have succeeded: Medial frontal negativity tracks failure in a social context. Psvchophvsiologv 48, 973-979.

Bush, G., Luu, P., \& Posner, M. I. (2000). Cognitive and emotional influences in anterior cingulate cortex. Trends in Cognitive Sciences, 4, 215-222.

Casey, B. J., Tottenham, N., Liston, C., \& Durston, S. (2005). Imaging the developing brain: What have we learned about cognitive development? Trends in Cognitive Sciences, 9, 104-110.

Casey, B. J., Trainor, R., Giedd, J., Vauss, Y., Vaituzis, C. K., Hamburger, S., et al. (1997). The role of the anterior cingulate in automatic and controlled processes: A developmental neuroanatomical study. Developmental Psvchobiologv, 3, 61-69. 
Chen, J., Wu, Y., Tong, G., Guan, X., \& Zhou, X. (2012). ERP correlates of social conformity in a line judgment task. $B M C$ Neuroscience, 13, 43.

Cohen, M. X., \& Ranganath, C. (2007). Reinforcement learning signals predict future decisions. Iournal of Neuroscience, 27, 371-378.

Crone, E. A., Somsen, R. J. M., Zanolie, K., \& van der Molen, M. W. (2006). A heart rate analysis of developmental change in feedback processing and rule shifting from childhood to early adulthood. Journal of Experimental Child Psychology, 95, 99-116.

Crone, E. A., Zanolie, K., van Leijenhorst, L., Westenberg, P. M., \& Rombouts, S. A. R. B. (2008). Neural mechanisms supporting flexible performance adjustment during development. Cognitive. Affective. \& Behavioral Neuroscience, 8, 165-177.

Csibra, G., Kushnerenko, E., \& Grossmann, T. (2008). Electrophysiological methods in studying infant cognitive development. In C. A. Nelson \& M. Luciana (Eds.), Handbook of developmental cognitive neuroscience (2nd ed., pp. 247-262). Cambridge, MA: MIT Press.

De Haan, M. (2007). Infant EEG and event-related potentials. Hove, England: Psychology Press.

Debener, S., Ullsperger, M., Siegel, M., Fiehler, K., von Cramon, D. Y., \& Engel, A. K. (2005). Trial-by-trial coupling of concurrent electroencephalogram and functional magnetic resonance imaging identifies the dynamics of performance monitoring. Journal of Neuroscience, 25, 11730-11737.

Folstein, J. R., \& van Petten, C. (2008). Influence of cognitive control and mismatch on the N2 component of the ERP: A review. Psychophysiology, 45, 152-170.

Fukushima, H., \& Hiraki, K. (2009). Whose loss is it? Human electrophysiological correlates of non-self reward processing Social Neuroscience, 4, 261-275.

Gehring, W. J., \& Willoughby, A. R. (2002). The medial frontal cortex and the rapid processing of monetary gains and losses Science, 295, 2279-2282.

Gogtay, N., Giedd, J. N., Lusk, L., Hayashi, K. M., Greenstein, D., Vaituzis, A. C., et al. (2004). Dynamic mapping of human cortical development during childhood through early adulthood. Proceedings of the National Academv of Sciences. U.S.A., 101, 8174-8179.

Hajcak, G., Moser, J. S., Holroyd, C. B., \& Simons, R. F. (2006) The feedback-related negativity reflects the binary evaluation of good versus bad outcomes. Biological Psychology, 71, 148-154.

Hämmerer, D., Li, S., Müller, V., \& Lindenberger, U. (2010). Life span differences in electrophysiological correlates of monitoring gains and losses during probabilistic reinforcement learning. Journal of Cognitive Neuroscience, 23, 579-592.

Hoehl, S., \& Wahl, S. (2012). Recording infant ERP data for cognitive research. Developmental Neuropsychology, 37, 187-209.

Holroyd, C. (2004). A note on the oddball N200 and the feedback ERN. Neurophysiology, 78, 447-455.

Holroyd, C. B., \& Coles, M. G. H. (2002). The neural basis of human error processing: Reinforcement learning, dopamine, and the error-related negativity. Psvchological Review, 109, 679-709.

Holroyd, C. B., Hajcak, G., \& Larsen, J. T. (2006). The good, the bad and the neutral: Electrophysiological responses to feedback stimuli. Brain Research, 1105, 93-101.

Holroyd, C. B., \& Krigolson, O. E. (2007). Reward prediction error signals associated with a modified time estimation task. Psychophysiologv, 44, 913-917.

Holroyd, C. B., Nieuwenhuis, S., Yeung, N., Nystrom, L., Mars, R. B., Coles, M. G. H., et al. (2004). Dorsal anterior cingulate cortex shows fMRI response to internal and external error signals. Nature Neuroscience, 7, 497-498.
Holroyd, C. B., Pakzad-Vaezi, K. L., \& Krigolson, O. E. (2008). The feedback correct-related positivity: Sensitivity of the event-related brain potential to unexpected positive feedback. Psvchotbvsiology, 45, 688-697.

Jia, S., Li, H., Luo, Y., Chen, A., Wang, B., \& Zhou, X. (2007). Detecting perceptual conflict by the feedback-related negativity in brain potentials. NeuroReport, 18, 1385-1388.

Kelly, A. C., Di Martino, A., Uddin, L. Q., Shehzad, Z., Gee, D. G., Reiss, P. T., et al. (2009). Development of anterior cingulate functional connectivity from late childhood to early adulthood. Cerebral Cortex, 19, 640-657.

Luo, Y., Sun, S., Mai, X., Gu, R., \& Zhang, H. (2011). Outcome evaluation in decision making: ERP studies. In S. Han \& E. Popper (Eds.), Culture and neural frames of cognition and communication (pp. 249-285). Heidelberg, Germany: Springer-Verlag.

Mai, X., Tardif, T., Doan, S. N., Liu, C., Gehring, W. J., \& Luo, Y. (2011). Brain activity elicited by positive and negative feedback in preschool-aged children. PLoS One, 6, e18774.

Marco-Pellarés, J., Cucurell, D., Münte, T. F., Strien, N., \& Rodriguez-Fornelis, A. (2011). On the number of trials needed for a stable feedback-related negativity. Psvchophysiology, 48, 852-860.

Mathewson, K. J., Dywan, J., Snyder, P. J., Tays, W. J., \& Segalowitz, S. J. (2008). Aging and electrocortical response to error feedback during a spatial learning task. Psvchobbusiology. 45, 936-948.

Miltner, W. H. R., Braun, C. H., \& Coles, M. G. H. (1997). Event-related brain potentials following incorrect feedback in a time-estimation task: Evidence for a "generic" neural system for error detection. Journal of Cognitive Neuroscience, 9, 788-798.

Müller, S. V., Möller, J., Rodriguez-Fornells, A., \& Münte, T. F. (2005). Brain potentials related to self-generated and external information used for performance monitoring. Clinical Neurophysiology, 116, 63-74.

Nelson, C. A., \& McCleery, J. P. (2008). Use of event-related potentials in the study of typical and atypical development. Journal of the American Academv of Child and Adolescent Psvchiatry, 47, 1252-1261.

Ridderinkhof, K. R., Ullsperger, M., Crone, E. A., \& Nieuwenhuis, S. (2004). The role of the medial frontal cortex in cognitive control. Science, 306, 443-447.

Rueda, M. R., Posner, M. I., \& Rothbart, M. K. (2004). Attentional control and self-regulation. In R. F. Baumeister \& K. D. Vohs (Eds.), Handbook of self-regulation: Research, theory, and applications (pp. 283-300). New York: Guilford Press.

Santesso, D. L., Dzyundzyak, A., \& Segalowitz, S. J. (2011). Age, sex and individual differences in punishment sensitivity: Factors influencing the feedback-related negativity. Psvchopbusiology, 48, 1481-1488.

Segalowitz, S. J., Santesso, D. L., Wiloughby, T., Reker, D. L., Campbell, K., Chalmers, H., et al. (2012). Adolescent peer interaction and trait surgency weaken medial prefrontal cortex responses to failure. Social Cognitive and Affective Neuroscience, 7, 115-124.

Tucker, D. M., Luu, P., Frishkoff, G., Quiring, J., \& Poulsen, C. (2003). Frontolimbic response to negative feedback in clinical depression. Iournal of Abnormal Psvchologv, 112, 667-678.

van Noordt, S. J., \& Segalowitz, S. J. (2012). Performance monitoring and the medial prefrontal cortex: A review of individual differences and context effects as a window on self-regulation. Frontiers in Human Neuroscience, 6, article 197.

Walsh, M. M., \& Anderson, J. R. (2012). Learning from experience: Event-related potential correlates of reward processing, neural adaptation, and behavioral choice. Neuroscience and Biobehavioral Reviews. 36, 1870-1884. 